\title{
On axisymmetrical boundary problem of unsteady motion of micropolar fluid in the half-space
}

\author{
I.H. EL-SIRAFY and A.W. ABOUTAHOUN* \\ Department of Mathematics, Faculty of Science, Alexandria University, Alexandria, Egypt
}

\begin{abstract}
The aim of this paper is developing an exact solution for the problem of axisymmetrical flow of unsteady motion of micropolar fluid in the half-space when the shear stresses are given on the boundary. The Laplace-Hankel transform technique is used to solve this problem. Some physical quantities such as velocities, pressure and microrotations are obtained and illustrated numerically.
\end{abstract}

Key words: micropolar fluid, axisymmetrical motion, Laplace-Hankel transform.

\section{Introduction}

The Newtonian relationships cannot be characterized to explain the behaviour of materials in shear. Eringen [1] proposed the basics of the theory of micropolar fluids display the effects of couple stresses, body couples and local rotary inertia. This theory might serve as a satisfactory model for describing the flow properties of polymeric fluids, liquid crystals which are made up of dumbbell molecules, animal blood and fluids containing certain additives.

El-Sirafy [3] generalized the results of the solution of the homogeneous Navier-Stokes equations in the half-plane for the slow motion of viscous incompressible fluids to the class of the micropolar fluids for the case of the given shear stresses on the boundary. El-Sirafy and Abdel-Moneim [4] investigated the unsteady two-dimensional motion of micropolar fluid within the half-plane due to the sudden motion of its horizontal boundary. Gorla et al. [5] presented a study on the effects of buoyancy on forced convection on an axisymmetric stagnation flow of micropolar fluids over a vertical cylinder with constant or linear variation of surface heat flux conditions. Faltas et al. [7] considered the problem of the steadystate axisymmetric flow of an incompressible micropolar fluid past two spherical particles. Under the Stokes flow approximation, they obtained a general solution which is constructed using superposition of the basic solutions in two moving spherical coordinate systems based on the centers of the particles. Lok et al. [8] studied the unsteady mixed convection boundary-layer flow of a micropolar fluid near the region of the stagnation point on a double-infinite vertical flat plate. Extensive reviews for the mathematical theory of equations and applications of micropolar fluids can be found in books by Łukaszewicz [10] and Eringen [2].

The aim of this paper is developing an exact solution for the problem of axisymmetrical flow of unsteady micropolar fluid in the half-space when the shear stresses are given on the boundary. The Laplace-Hankel transform technique is used to solve this problem. Some physical quantities such as veloc- ities, microrotation and pressure are obtained and illustrated numerically. This boundary problem could be met in the study of the flow near a solid boundary which is not wet by the given micropolar fluid.

\begin{tabular}{|c|c|}
\hline \multicolumn{2}{|c|}{ Nomenclature } \\
\hline$H(t)$ & Heaviside function \\
\hline$p$ & pressure \\
\hline$t$ & time ................ $\mathrm{s}$ \\
\hline$\rho$ & fluid density $\ldots \ldots \ldots \ldots \ldots . . . \mathrm{kg} \mathrm{m}^{-3}$ \\
\hline $\begin{array}{l}u(r, y, t) \\
v(r, y, t)\end{array}$ & $\begin{array}{l}\text { horizontal and normal components of the velocity along } \\
\text { the } r \text { - and } y \text {-directions, respectively } \ldots \ldots \ldots \ldots \ldots \ldots \mathrm{m} \mathrm{s}^{-1}\end{array}$ \\
\hline$\gamma$ & spin gradient viscosity $\ldots \ldots \ldots \ldots \ldots . . . . . . . . k g ~ m s^{-1}$ \\
\hline$\mu$ & classical shear viscosity coefficient $\ldots \ldots \ldots \ldots \ldots \ldots \mathrm{kg} \mathrm{m}^{-1} \mathrm{~s}^{-1}$ \\
\hline$\kappa$ & vortex viscosity $\ldots \ldots \ldots \ldots \ldots . . . . \mathrm{kg} \mathrm{m}^{-1} \mathrm{~s}^{-1}$ \\
\hline$j$ & microinertia per unit mass ................ $\mathrm{m}^{2}$ \\
\hline$\lambda$ & second order viscosity coefficient \\
\hline$\alpha, \beta$ & viscosity coefficients \\
\hline$l$ & micropolar length parameter ................. m \\
\hline$n$ & microrotation $\ldots \ldots \ldots \ldots \ldots . . . \mathrm{s}^{-1}$ \\
\hline$\widehat{y r}, \widehat{r y}$ & shear stresses \\
\hline$\delta(t)$ & Dirac delta function of $t$ \\
\hline
\end{tabular}

The dimensions considered in Nomenclature are presented in Lok et al. [8].

The rest of this paper is organized as follows: Sec. 2 presents the problem formulation. A solution methodology is developed in Sec. 3. A numerical results is presented in Sec. 4. A conclusion for this work is presented at the end in Sec. 5.

\section{Problem formulation}

The field equations of the micropolar fluid given by Eringen [1] are as follows:

Conservation of mass

$$
\frac{\partial \rho}{\partial t}+\nabla \cdot \rho \vec{q}=0
$$

\footnotetext{
*e-mail: tahoun44@yahoo.com
} 
I.H. El-Sirafy and A.W. Aboutahoun

Balance of momentum

$$
\begin{gathered}
\nabla p=(\lambda+2 \mu+\kappa) \nabla(\nabla \cdot \vec{q})+\rho \vec{f} \\
-(\mu+\kappa) \nabla \times(\nabla \times \vec{q})-\rho \frac{\partial \vec{q}}{\partial t}+\kappa \nabla \times \vec{N} .
\end{gathered}
$$

Balance of angular momentum

$$
\begin{gathered}
\rho j \frac{\partial \vec{N}}{\partial t}=(\alpha+\beta+\gamma) \nabla(\nabla \cdot \vec{N})-\gamma \nabla \times(\nabla \times \vec{N}) \\
+\kappa \nabla \times \vec{q}-2 \kappa \vec{N}+\rho \vec{g},
\end{gathered}
$$

where $\rho$ is the fluid density, $\vec{N}=\left(N_{1}, N_{2}, N_{3}\right)$ is the microrotation, $\vec{q}=\left(q_{1}, q_{2}, q_{3}\right)$ is the velocity, $\vec{f}=\left(f_{1}, f_{2}, f_{3}\right)$ is the body force per unit mass, and $\vec{g}=\left(g_{1}, g_{2}, g_{3}\right)$ is the body couple per unit mass. The material's constants $\lambda$ and $\mu$ are the classical viscosity coefficients, and $\alpha, \beta, \gamma$ and $\kappa$ are the viscosity coefficients for micropolar fluids.

Neglecting the thermal effect the material constants $\alpha, \beta$, $\gamma, \lambda, \mu, \kappa$ have to satisfy the following inequalities

$$
\begin{array}{lrr}
2 \mu+\kappa \geq 0, & \kappa \geq 0, & 3 \lambda+2 \mu+\kappa \geq 0 \\
\gamma \geq 0, & \gamma \geq|\beta|, & 3 \alpha+\beta+\gamma \geq 0
\end{array}
$$

The problem under consideration we confine ourselves for studying equations governing the flow of an incompressible micropolar fluid in the absence of body force and body couple are given by

$$
\begin{gathered}
\nabla \cdot \vec{q}=0, \\
\nabla p=-(\mu+\kappa) \nabla \times(\nabla \times \vec{q})-\rho \frac{\partial \vec{q}}{\partial t}+\kappa \nabla \times \vec{N}, \\
\rho j \frac{\partial \vec{N}}{\partial t}=(\alpha+\beta+\gamma) \nabla(\nabla \cdot \vec{N}) \\
-\gamma \nabla \times(\nabla \times \vec{N})+\kappa \nabla \times \vec{q}-2 \kappa \vec{N} .
\end{gathered}
$$

This model contains as a special case the classical model of Navier and Stokes. In case of $\alpha=\beta=\gamma=\kappa=0$, microrotation $\vec{N}$ becomes zero. Also note that for $\kappa=0$, the velocity $\vec{q}$ and the microrotation $\vec{N}$ are uncoupled and the global motion is unaffected by the microrotations (see, Lukaszewicz [10], Eringen [2]). Hence, Eq. (5) reduces to the Navier-Stokes equations which takes the form

$$
\nabla p=-\mu \nabla \times(\nabla \times \vec{q})-\rho \frac{\partial \vec{q}}{\partial t} .
$$

Since $\nabla \times(\nabla \times \vec{q})=\nabla(\nabla \cdot \vec{q})-\nabla^{2} \vec{q}$, then Eq. (7) takes the form

$$
\nabla p=\mu \nabla^{2} \vec{q}-\rho \frac{\partial \vec{q}}{\partial t} .
$$

Equations (4) and (8) represent the Navier-Stokes equations. The linearized Navier-Stokes equations of twodimensional unsteady motion of an incompressible fluid in the upper half-plane $(-\infty<x<\infty, y>0 \mid t>0)$ are the same Eqs. (4) and (8). But in this case, $\vec{q}=$ $(u(x, y, t), v(x, y, t), 0)$, where $u(x, y, t)$ and $v(x, y, t)$ are the tangential and normal components of the velocity respectively. These are the Navier-Stokes equations in Cartestian coordinates for the considered problem.
For the problem under consideration, the suitable coordinates will be cylindrical polar coordinate system $(r, \theta, y)$. Cylindrical coordinates are chosen to take advantage of symmetry, so that a velocity component can disappear. Axisymmetric flow with the assumption of no tangential velocity and the remaining quantities are independent of $\theta$. The $y$-axis is taken perpendicular to the bounding $z x$-plane as the axis of symmetry. The flow components is shown schematically in Fig. 1. For the axisymmetrical motion of an incompressible micropolar fluid, the velocity and microrotation vectors are taken the form

$$
\begin{gathered}
\vec{q}=(u(r, y, t), 0, v(r, y, t)), \\
\vec{N}=(0, n(r, y, t), 0),
\end{gathered}
$$

where the velocity components $u$ and $v$ are taken to be in the direction of $r$ and $y$ axes, respectively.

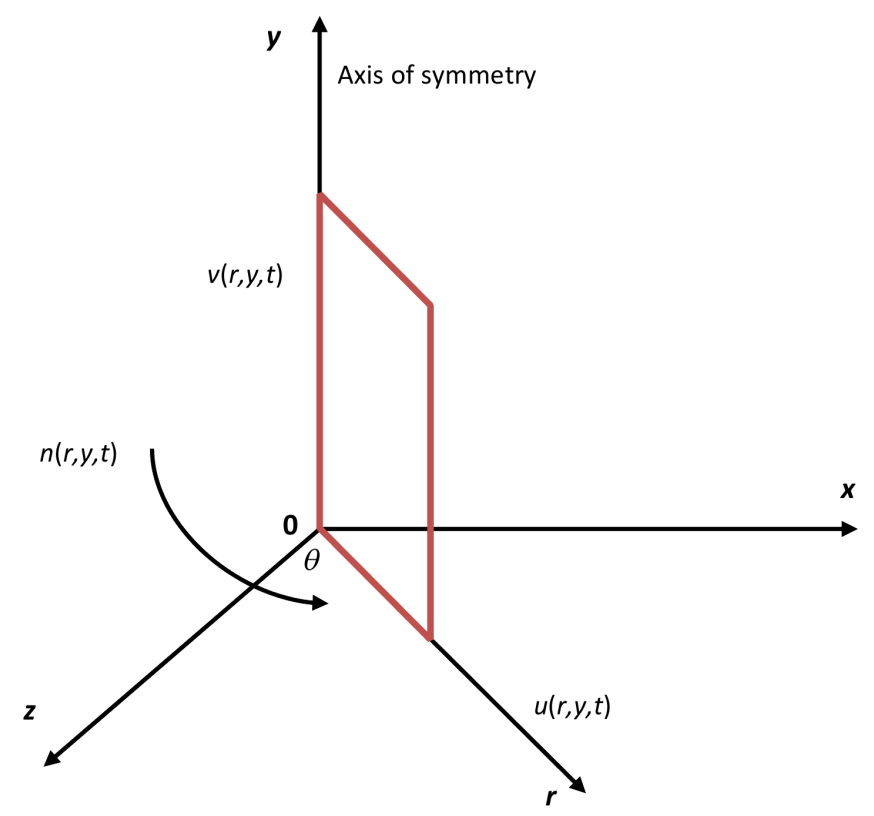

Fig. 1. Physical model and coordinate system

In the absence of both external forces and body couples the linearized equations of axisymmetrical motion of an incompressible micropolar fluid in the upper half-space $(0 \leq r<\infty, y>0 \mid t>0)$ are

$$
\begin{gathered}
\frac{\partial p}{\partial r}=(\mu+\kappa) \triangle^{*} u-\rho \frac{\partial u}{\partial t}+\kappa \frac{\partial n}{\partial y}, \\
\frac{\partial p}{\partial y}=(\mu+\kappa) \triangle v-\rho \frac{\partial v}{\partial t}-\frac{\kappa}{r} \frac{\partial}{\partial r}(r n), \\
\rho j \frac{\partial n}{\partial t}=\gamma \triangle^{*} n-2 \kappa n+\kappa\left(\frac{\partial v}{\partial r}-\frac{\partial u}{\partial y}\right),
\end{gathered}
$$

where

$$
\triangle^{*}=\triangle-\frac{1}{r^{2}}, \quad \triangle=\frac{\partial^{2}}{\partial r^{2}}+\frac{1}{r} \frac{\partial}{\partial r}+\frac{\partial^{2}}{\partial y^{2}} .
$$

The equation of continuity is

$$
\frac{1}{r} \frac{\partial}{\partial r}(r u)+\frac{\partial v}{\partial y}=0
$$


On axisymmetrical boundary problem of unsteady motion of micropolar fluid in the half-space

where $\gamma, \mu, \kappa, j$ are viscosity coefficients, $p(r, y)$ is the pressure and the components of the shear stresses $\widehat{y r}$ and $\widehat{r y}$ are given by

$$
\left.\begin{array}{l}
\widehat{y r}=(\mu+\kappa) \frac{\partial u}{\partial y}+\mu \frac{\partial v}{\partial r}+\kappa n, \\
\widehat{r y}=(\mu+\kappa) \frac{\partial v}{\partial r}+\mu \frac{\partial u}{\partial y}-\kappa n
\end{array}\right\} .
$$

The boundary conditions are

$$
\begin{gathered}
v(r, 0, t)=0, \\
n(r, 0, t)=0, \\
\left.\frac{\partial u}{\partial y}\right|_{y=0}=\frac{\left.\widehat{y r}\right|_{y=0}}{\mu+\kappa}=\frac{\left.\widehat{r y}\right|_{y=0}}{\mu}=\frac{\partial G(r, t)}{\partial r}, \\
u(r, \infty, t)=v(r, \infty, t)=n(r, \infty, t)=0,
\end{gathered}
$$

where $G(r, t)$ is a given function.

The initial conditions are

$$
u(r, y, 0)=v(r, y, 0)=n(r, y, 0)=0 .
$$

\section{Solution methodology}

Introduce two real functions $\varphi(r, y, t)$ and $\psi(r, y, t)$ such that

$$
\begin{gathered}
u(r, y, t)=\frac{\partial \varphi}{\partial r}+\frac{\partial \psi}{\partial y}, \\
v(r, y, t)=\frac{\partial \varphi}{\partial y}-\frac{1}{r} \frac{\partial}{\partial r}(r \psi) .
\end{gathered}
$$

The equation of continuity (12) gives

$$
\triangle \varphi=0 .
$$

Substituting from (16) in the system (9)-(11) and observing that

$$
\begin{gathered}
\triangle^{*} \frac{\partial \varphi}{\partial r}=\frac{\partial}{\partial r} \triangle \varphi \\
\triangle\left[\frac{1}{r} \frac{\partial}{\partial r}(r \psi)\right]=\frac{1}{r} \frac{\partial}{\partial r}\left[r \triangle^{*} \psi\right] .
\end{gathered}
$$

We can write

$$
\begin{gathered}
\frac{\partial}{\partial y}\left[p-(\mu+\kappa) \Delta \varphi+\rho \frac{\partial \varphi}{\partial t}\right] \\
=-\frac{1}{r} \frac{\partial}{\partial r}\left[r\left((\mu+\kappa) \Delta^{*} \psi-\rho \frac{\partial \psi}{\partial t}+\kappa n\right)\right], \\
\frac{\partial}{\partial r}\left[p-(\mu+\kappa) \triangle \varphi+\rho \frac{\partial \varphi}{\partial t}\right] \\
=\frac{\partial}{\partial y}\left[(\mu+\kappa) \triangle^{*} \psi-\rho \frac{\partial \psi}{\partial t}+\kappa n\right] .
\end{gathered}
$$

Thus the system (9)-(12) is equivalent to

$$
\begin{gathered}
p=-\rho \frac{\partial \varphi}{\partial t}, \\
\triangle \varphi=0 \\
(\mu+\kappa) \triangle^{*} \psi-\rho \frac{\partial \psi}{\partial t}+\kappa n=0,
\end{gathered}
$$

$$
\kappa \triangle^{*} \psi+\rho j \frac{\partial n}{\partial t}+2 \kappa n-\gamma \triangle^{*} n=0 .
$$

Using the technique of Laplace-Hankel transform

$$
\begin{gathered}
\widetilde{\varphi}(q, y, \lambda)=\int_{0}^{\infty} e^{-\lambda t} \int_{0}^{\infty} \varphi(r, y, t) r J_{0}(q r) d r d t, \\
\varphi(r, y, t)=\frac{1}{2 \pi i} \int_{\sigma-i \infty}^{\sigma+i \infty} e^{\lambda t} \int_{0}^{\infty} \widetilde{\varphi}(q, y, \lambda) q J_{0}(q r) d q d \lambda, \\
\widetilde{\psi}(q, y, \lambda)=\int_{0}^{\infty} e^{-\lambda t} \int_{0}^{\infty} \psi(r, y, t) r J_{1}(q r) d r d t, \\
\psi(r, y, t)=\frac{1}{2 \pi i} \int_{\sigma-i \infty}^{\sigma+i \infty} e^{\lambda t} \int_{0}^{\infty} \widetilde{\psi}(q, y, \lambda) q J_{1}(q r) d q d \lambda, \\
\widetilde{n}(q, y, \lambda)=\int_{0}^{\infty} e^{-\lambda t} \int_{0}^{\infty} n(r, y, t) r J_{1}(q r) d r d t, \\
n(r, y, t)=\frac{1}{2 \pi i} \int_{\sigma-i \infty}^{\sigma+i \infty} e^{\lambda t} \int_{0}^{\infty} \widetilde{n}(q, y, \lambda) q J_{1}(q r) d q d \lambda, \\
G(r, t)=\frac{1}{2 \pi i} \int_{\sigma-i \infty}^{\infty} e^{\lambda t} \int_{0}^{\infty} \widetilde{G}(q, \lambda) q J_{0}(q r) d q d \lambda, \\
\widetilde{G}(q, \lambda)=\int_{0}^{\infty-\lambda t} \int_{0}^{\infty} G(r, t) r J_{0}(q r) d r d t .
\end{gathered}
$$

and knowing that

$$
\begin{gathered}
-J_{1}(r q)=\frac{1}{q} \frac{\partial}{\partial r}\left[J_{0}(r q)\right], \\
\frac{d}{d y}\left[y J_{1}(y)\right]=y J_{0}(y), \\
\int_{0}^{\infty} e^{-\lambda t} \int_{0}^{\infty}[\Delta \varphi(r, y, t)] r J_{0}(q r) d r d t \\
=\widetilde{\varphi}^{\prime \prime}-q^{2} \widetilde{\varphi}(q, y, \lambda), \\
\int_{0}^{\infty} e^{-\lambda t} \int_{0}^{\infty}\left[\triangle^{*} \psi(r, y, t)\right] r J_{1}(q r) d r d t \\
=\widetilde{\psi}^{\prime \prime}-q^{2} \widetilde{\psi}(q, y, \lambda) .
\end{gathered}
$$

The system (21)-(23) become

$$
\begin{gathered}
\widetilde{\varphi}^{\prime \prime}-q^{2} \widetilde{\varphi}=0, \\
(\mu+\kappa) \widetilde{\psi}^{\prime \prime}-\left[(\mu+\kappa) q^{2}+\rho \lambda\right] \widetilde{\psi}+\kappa \widetilde{n}=0, \\
\gamma \widetilde{n}^{\prime \prime}-\left(\gamma q^{2}+2 \kappa+\rho j \lambda\right) \widetilde{n}-\kappa \widetilde{\psi}^{\prime \prime}+\kappa q^{2} \widetilde{\psi}=0 .
\end{gathered}
$$

Now we may take as a trial solution

$$
\widetilde{\psi}=A e^{-\zeta y}, \quad \widetilde{n}=B e^{-\zeta y}, \quad \widetilde{\varphi}=C e^{-q y} .
$$


I.H. El-Sirafy and A.W. Aboutahoun

By substituting from (31) into (29), (30), we get

$$
\begin{gathered}
{\left[(\mu+\kappa)\left(\zeta^{2}-q^{2}\right)-\rho \lambda\right] A+\kappa B=0,} \\
-\kappa\left(\zeta^{2}-q^{2}\right) A+\left[\gamma\left(\zeta^{2}-q^{2}\right)-(2 \kappa+\rho j \lambda)\right] B=0 .
\end{gathered}
$$

Then the condition for a nonzero solution is

$$
\begin{gathered}
\gamma\left(\zeta^{2}-q^{2}\right)^{2}-\left(2 \kappa+\rho j \lambda+\frac{\rho \lambda \gamma}{\mu+\kappa}\right)\left(\zeta^{2}-q^{2}\right) \\
+\rho \lambda\left(\frac{2 \kappa+\rho j \lambda}{\mu+\kappa}\right)=0 .
\end{gathered}
$$

Here for the simplification we confine our study to a special class of fluids in which the spin gradient viscosity $j$ is assumed to be a constant and given by

$$
j=\frac{2 \gamma}{2 \mu+\kappa} \text {. }
$$

This latter relation is imposed to allow the field equations to predict the correct behavior in the limiting case when microstructure effects become negligible and microrotation reduces to the angular velocity. Many authors have assumed that special class of fluids, see Kline and Allen [9], Ahmadi [11], Allen and Kline [12], and Rees and Bassom [13].

Consider the parameters

$$
l=\sqrt{\frac{\gamma}{2(2 \mu+\kappa)}}=\frac{\sqrt{j}}{2}, \quad M=\sqrt{\frac{\kappa}{2 \mu+\kappa}} .
$$

The parameter $l$ measures the relative length of the fluid microstructure. The value of $l$ reflects the microscopic properties of the fluid. The parameter $M, 0 \leq M \leq 1$ characterizes coupling between the vortex viscosity coefficient $\kappa$ and the shear viscosity coefficient $\mu$. For more details, see [14].

If the value of the parameter $M \rightarrow 0$, then the equations of momentum (5) and angular momentum (6) become independent of each other and the first one transforms into a classical Navier-Stokes equation for Newtonian fluid [14].

The roots of Eq. (32) are given by

$$
\zeta_{1}^{2}=q^{2}+a \rho \lambda, \quad \zeta_{2}^{2}=q^{2}+b \rho \lambda+c,
$$

where

$$
a=\frac{2}{2 \mu+\kappa}, \quad b=\frac{1}{\mu+\kappa}, \quad c=\frac{2 b \kappa}{a \gamma} .
$$

The boundary conditions (14) become

$$
\begin{gathered}
\left.\frac{d \widetilde{\varphi}}{d y}\right|_{y=0}-q \widetilde{\psi}(q, 0, \lambda)=0, \\
\left.\frac{d^{2} \widetilde{\psi}}{d y^{2}}\right|_{y=0}-\left.q \frac{d \widetilde{\varphi}}{d y}\right|_{y=0}=-q \widetilde{G}(q, \lambda), \\
\tilde{n}(q, 0, \lambda)=0 .
\end{gathered}
$$

Thus, after some calculations we obtain

$$
\begin{gathered}
\widetilde{\varphi}(q, y, \lambda)=q \frac{\widetilde{G}(q, \lambda)}{b \rho \lambda} e^{-q y}, \\
\widetilde{\psi}(q, y, \lambda)=-\frac{1}{b}\left[q \frac{\widetilde{G}(q, \lambda)}{\rho \lambda} e^{-\zeta_{1} y}+\frac{\kappa b^{2}}{c} \widetilde{n}(q, y, \lambda)\right],
\end{gathered}
$$

$$
\widetilde{n}(q, y, \lambda)=-\frac{c q \widetilde{G}(q, \lambda)}{\kappa b^{2}\left(\rho \lambda-c_{0}\right)}\left(e^{-\zeta_{1} y}-e^{-\zeta_{2} y}\right),
$$

where

$$
c_{0}=\frac{c}{a-b} .
$$

Assume that

$$
\begin{gathered}
G(r, t)=H_{0} H(t) H(\epsilon-r), \\
H(\epsilon-r)=\left\{\begin{array}{ll}
1 & 0<r \leq \epsilon \\
0 & r>\epsilon
\end{array},\right.
\end{gathered}
$$

where $\frac{\partial G(r, t)}{\partial r}=-H_{0} H(t) \delta(\epsilon-r), H(t)$ is the Heaviside function and $H_{0}$ is a constant.

Thus

$$
\widetilde{G}(q, \lambda)=\frac{H_{0} \epsilon J_{1}(q \epsilon)}{\lambda q} .
$$

Using Laplace-Hankel transform, we get after some calculation

$$
\varphi(r, y, t)=\frac{H_{0} \epsilon t}{b \rho} \int_{0}^{\infty} q e^{-q y} J_{0}(q r) J_{1}(q \epsilon) d q,
$$

$$
\begin{gathered}
n(r, y, t)=\frac{H_{0} \epsilon c}{\kappa b^{2} c_{0}} \int_{0}^{\infty} q J_{1}(q r) J_{1}(q \epsilon)[M(y, t, q, a, 0,0) \\
-M(y, t, q, b, 0, c) \\
\left.-M\left(y, t, q, a, c_{0}, 0\right)+M\left(y, t, q, b, c_{0}, c\right)\right] d q, \\
\psi(r, y, t)=n_{0}(r, y, t)-\frac{\kappa b}{c} n(r, y, t),
\end{gathered}
$$

where

$$
\begin{gathered}
M(y, t, q, \alpha, \beta, \delta)=\frac{\rho}{2 \pi i} \int_{\sigma-i \infty}^{\sigma+i \infty} \frac{e^{\lambda t-\sqrt{q^{2}+\alpha \rho \lambda+\delta} y}}{\rho \lambda-\beta} d \lambda, \\
\sigma=\operatorname{Re} \lambda>0
\end{gathered}
$$

can be put in the form [6]:

$$
\begin{gathered}
M(y, t, q, \alpha, \beta, \delta)=M_{+}(y, t, q, \alpha, \beta, \delta) \\
+M_{-}(y, t, q, \alpha, \beta, \delta), \\
M_{ \pm}(y, t, q, \alpha, \beta, \delta)=\frac{1}{2} e^{ \pm \sqrt{q^{2}+\alpha \beta+\delta} y+\frac{\beta t}{\rho}} \\
\cdot \operatorname{erf} c\left(\frac{y}{2} \sqrt{\frac{\alpha \rho}{t}} \pm \sqrt{\left(q^{2}+\alpha \beta+\delta\right) \frac{t}{\alpha \rho}}\right), \\
n_{0}(r, y, t)=\frac{-H_{0} \epsilon}{b \rho} \int_{0}^{\infty} q J_{1}(q r) J_{1}(q \epsilon) P_{0}(y, t, q) d q,
\end{gathered}
$$

where

$$
\begin{gathered}
P_{0}(y, t, q)=\frac{1}{2 \pi i} \int_{\sigma-i \infty}^{\sigma+i \infty} \frac{e^{\lambda t-\sqrt{q^{2}+a \rho \lambda} y}}{\lambda^{2}} d \lambda \\
=\rho \lim _{\beta \rightarrow 0} \frac{\partial}{\partial \beta} M(y, t, q, a, \beta, 0) \\
=P_{+}(y, t, q)+P_{-}(y, t, q),
\end{gathered}
$$


On axisymmetrical boundary problem of unsteady motion of micropolar fluid in the half-space

where

$P_{ \pm}(y, t, q)=\frac{\rho}{2} e^{ \pm q y} \operatorname{erf} c\left(\frac{y}{2} \sqrt{\frac{a \rho}{t}} \pm q \sqrt{\frac{t}{a \rho}}\right)\left[\frac{t}{\rho} \pm \frac{y a}{2 q}\right]$.

To calculate the stream function, we obtain from (44)

$$
\frac{\partial \varphi}{\partial r}=\frac{H_{0} \epsilon t}{b \rho} \int_{0}^{\infty} q e^{-q y} \frac{\partial\left(J_{0}(q r)\right)}{\partial r} J_{1}(q \epsilon) d q
$$

where

$$
\varphi_{*}(r, y, t)=\frac{H_{0} \epsilon t}{b \rho} \int_{0}^{\infty} q e^{-q y} J_{1}(q r) J_{1}(q \epsilon) d q .
$$

Then

$$
u=\frac{\partial}{\partial y}\left(\psi+\varphi_{*}\right)
$$

Also,

$$
\frac{\partial \varphi(r, y, t)}{\partial y}=-\frac{H_{0} \epsilon t}{b \rho} \int_{0}^{\infty} q^{2} e^{-q y} J_{0}(q r) J_{1}(q \epsilon) d q .
$$

Using (25), we get

$$
\begin{gathered}
\frac{\partial \varphi}{\partial y}=-\frac{H_{0} \epsilon t}{b \rho} \int_{0}^{\infty} q \frac{e^{-q y}}{r} \frac{\partial\left(r J_{1}(q r)\right)}{\partial r} J_{1}(q \epsilon) d q \\
=-\frac{1}{r} \frac{\partial}{\partial r}\left[r \int_{0}^{\infty} \frac{H_{0} \epsilon t}{b \rho} q e^{-q y} J_{1}(q r) J_{1}(q \epsilon) d q\right] \\
=-\frac{1}{r} \frac{\partial}{\partial r}\left(r \varphi_{*}(r, y, t)\right)
\end{gathered}
$$

and from (16)

$$
v=-\frac{1}{r} \frac{\partial}{\partial r}\left[r\left(\psi+\varphi_{*}\right)\right] .
$$

Thus we can write

$$
u=\frac{\partial \Psi}{\partial y}, \quad v=-\frac{1}{r} \frac{\partial}{\partial r}(r \Psi)
$$

where the stream function $\Psi$ is given by

$$
\Psi=\psi(r, y, t)+\varphi_{*}(r, y, t) .
$$

From (20) and (44), the pressure is given by

$$
p(r, y)=-\frac{H_{0} \epsilon}{b} \int_{0}^{\infty} q e^{-q y} J_{0}(q r) J_{1}(q \epsilon) d q
$$

i.e., the pressure in this case is a function independent of $t$.

\section{Numerical results}

The horizontal velocity $u(r, y, t)$ given by Eq. (53), the vertical velocity $v(r, y, t)$ given by Eq. (54), the stream function $\Psi(r, y, t)$ given by Eq. (55), the microrotation $n(r, y, t)$ given by Eq. (45), the shear stresses $\widehat{y r}$ and $\widehat{r y}$ given by Eq. (13), and the pressure $p(r, y)$ given by Eq. (48) are implemented numerically for different values of $r, y, t$.

For the computational work, a Matlab and Mathcad program are used to invert the transform in order to obtain the results in the physical domain, the following material constants are considered

$$
\begin{gathered}
\kappa=0.1 \mathrm{~kg} \mathrm{~m}^{-1} \mathrm{~s}^{-1}, \quad \gamma=1.05 \mathrm{~kg} \mathrm{~m} \mathrm{~s}^{-1}, \\
\mu=0.05 \mathrm{~kg} \mathrm{~m}^{-1} \mathrm{~s}^{-1}, \quad \rho=1.05 \mathrm{~kg} \mathrm{~m}^{-3}, \\
\epsilon=0.05, \quad H_{0}=0.001 .
\end{gathered}
$$

Figures 2-5 illustrate the stream function $\Psi(r, y, t)$ with one of the independent parameters $r, y$, and $t$ when values are given for the other two variables. The variation of the horizontal velocity $u(r, y, t)$ versus one of the parameters $r, y, t$ for different values of the other two parameters are shown in the plots presented in Figs. 6-9. Figures 10-13 show the variation of the normal velocity $v(r, y, t)$ with one of the parameters $r, y, t$ for different values of the other two parameters. Figures 14-19 display the variation of the microrotation $n(r, y, t)$ versus one of the parameters $r, y, t$ for different values of the other two parameters. The variations of the shear stresses $\widehat{y r}$ and $\widehat{r y}$ with one of the parameters $r, y, t$ for different values of the other two parameters are depicted in Figs. 20-27. The pressure $p(r, y)$ does not depend on time and it depends only on $r$ and $t$. Figures 28-29 display the pressure versus $r$ and $y$.

Figures 30-39 are three dimensional plots to display the stream function, the velocity components, pressure, microrotation, and shear stresses with $r$ and $y$. Figures 30 and 31 display the stream function with different scales for $r$ and $y$. Similarly, Figs. 34 and 35 illustarte the pressure and Figs. 36 and 37 display the microrotation with different scales for $r$ and $y$.

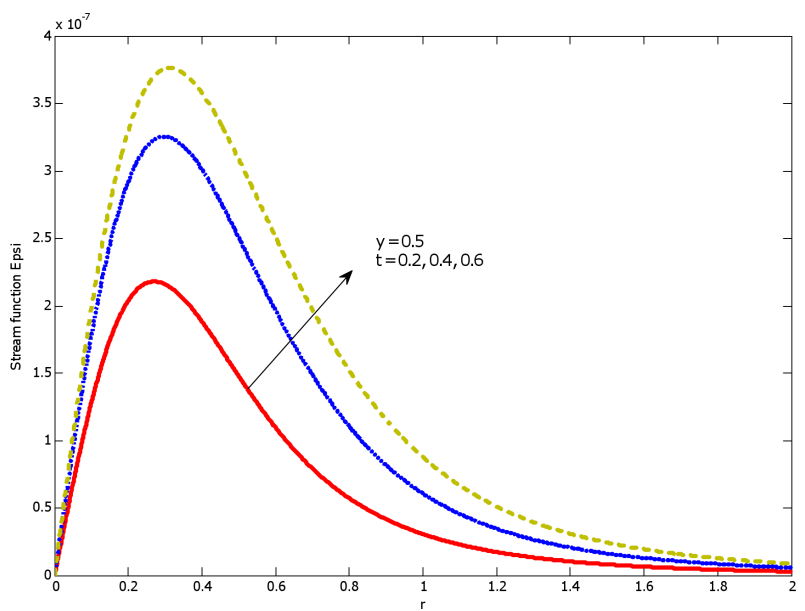

Fig. 2. Variation of the stream function $\Psi(r, y, t)$ with $r$

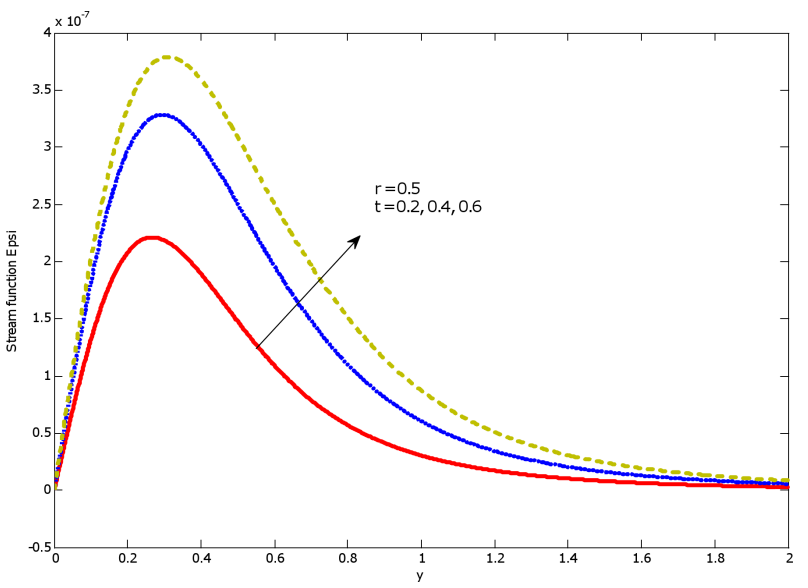

Fig. 3. Variation of the stream function $\Psi(r, y, t)$ with $y$ 
I.H. El-Sirafy and A.W. Aboutahoun

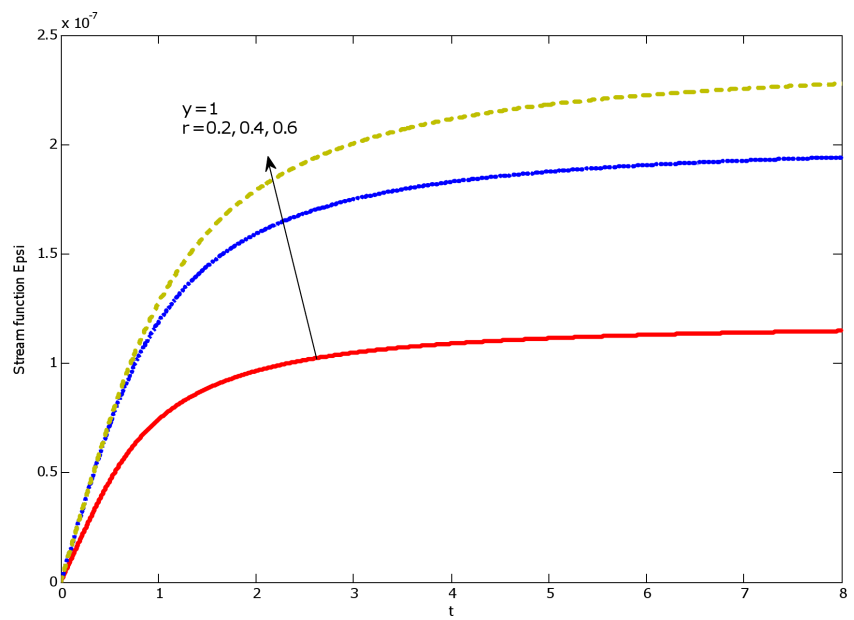

Fig. 4. Variation of the stream function $\Psi(r, y, t)$ with $t$

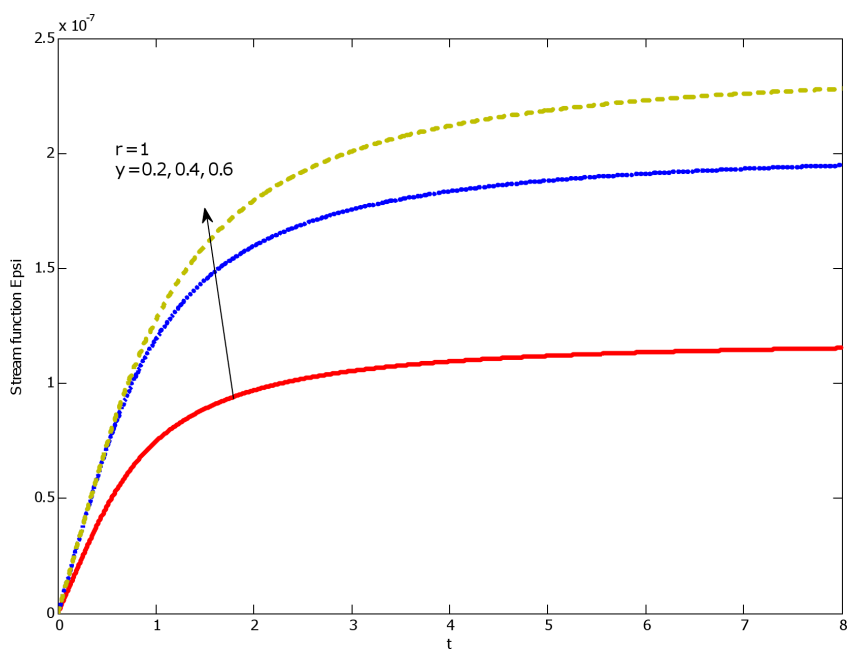

Fig. 5. Variation of the stream function $\Psi(r, y, t)$ with $t$

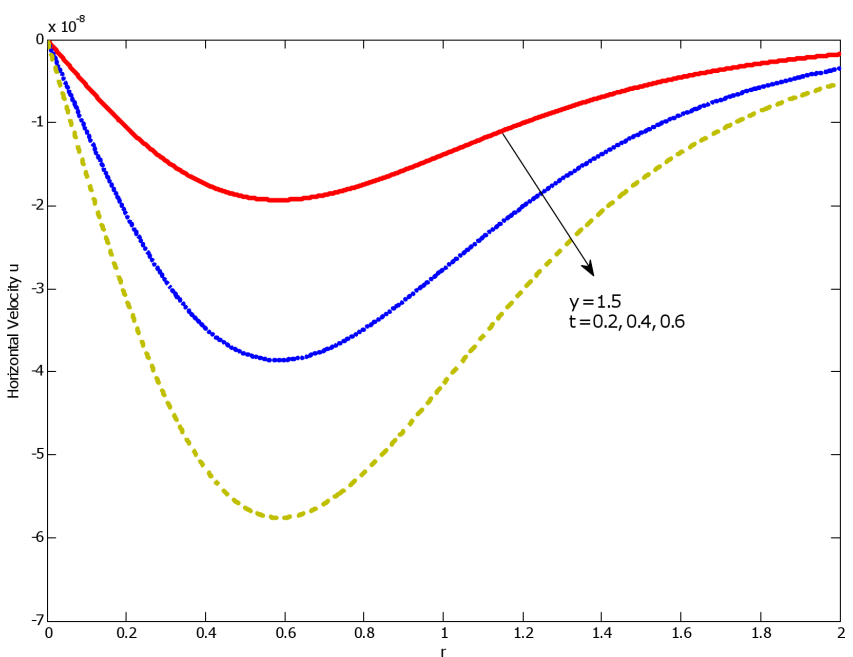

Fig. 6. Variation of the horizontal velocity $u(r, y, t)$ with $r$

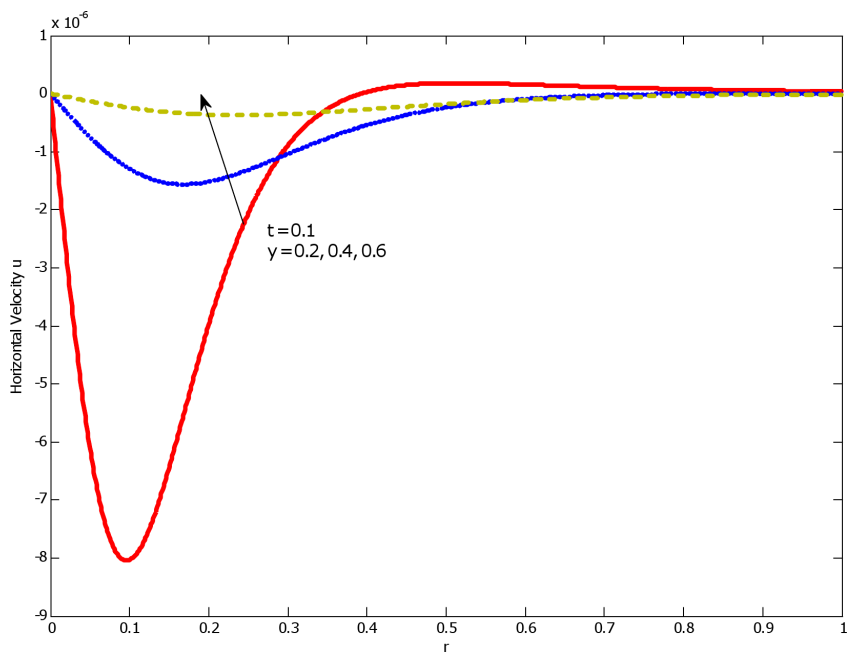

Fig. 7. Variation of the horizontal velocity $u(r, y, t)$ with $r$

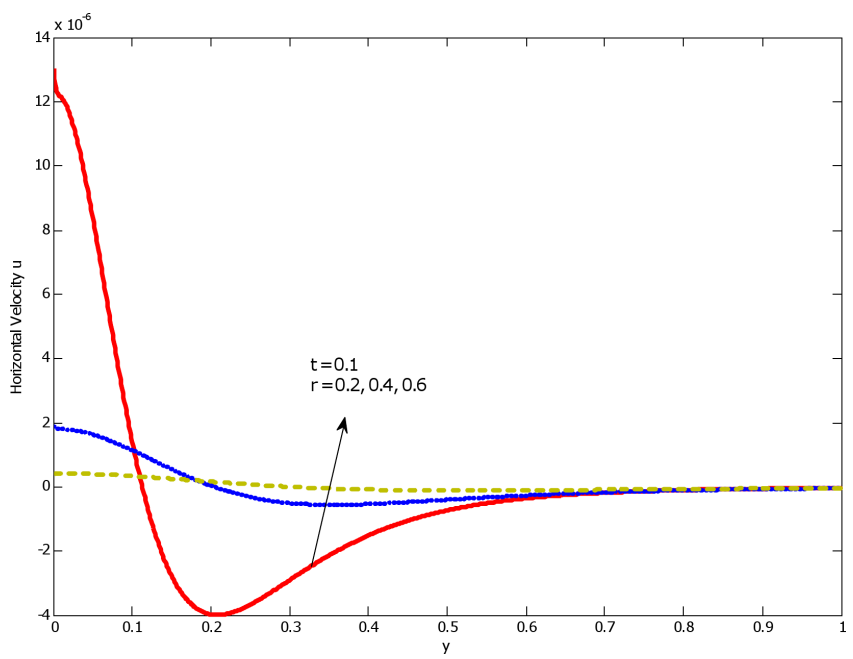

Fig. 8. Variation of the horizontal velocity $u(r, y, t)$ with $y$

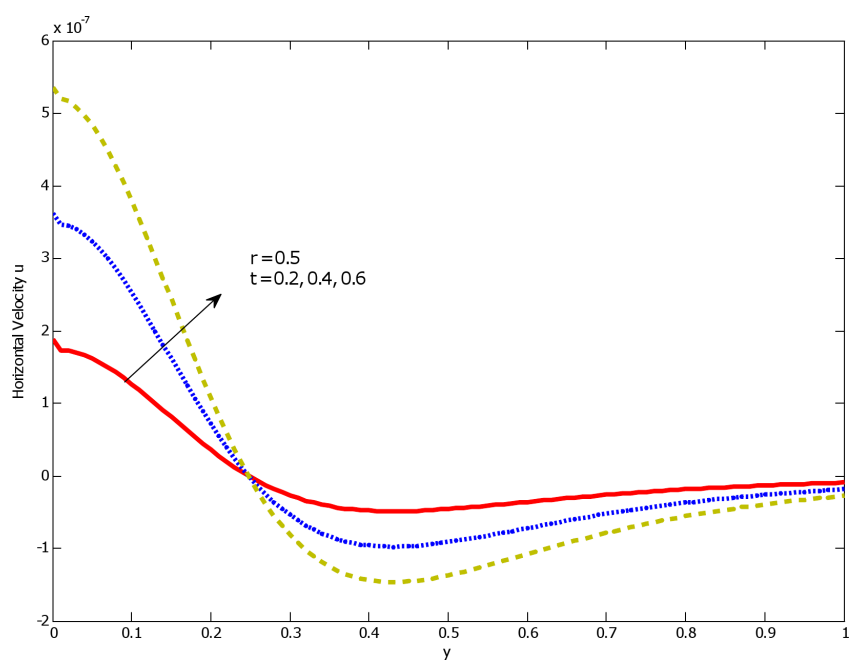

Fig. 9. Variation of the horizontal velocity $u(r, y, t)$ with $y$ 
On axisymmetrical boundary problem of unsteady motion of micropolar fluid in the half-space

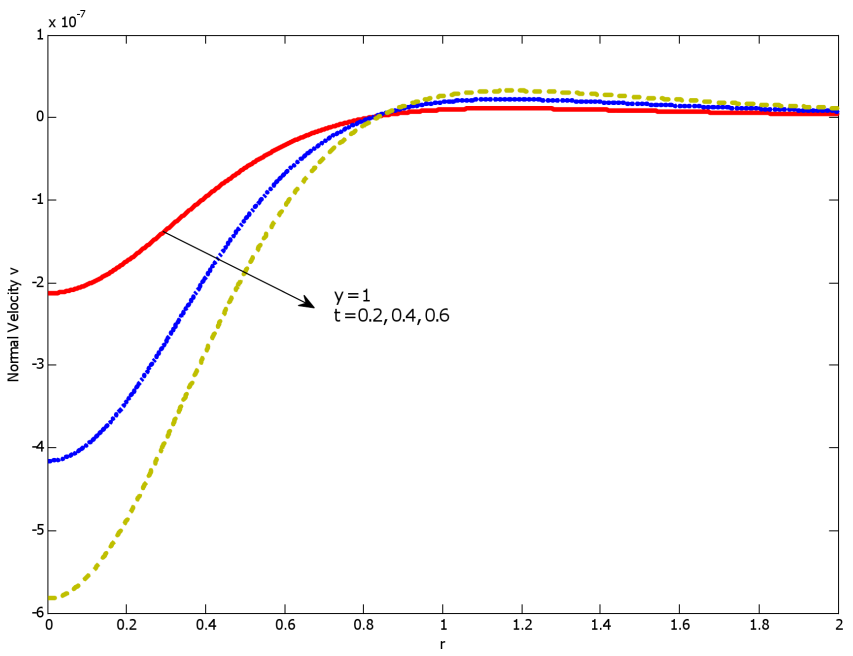

Fig. 10. Variation of the normal velocity $v(r, y, t)$ with $r$

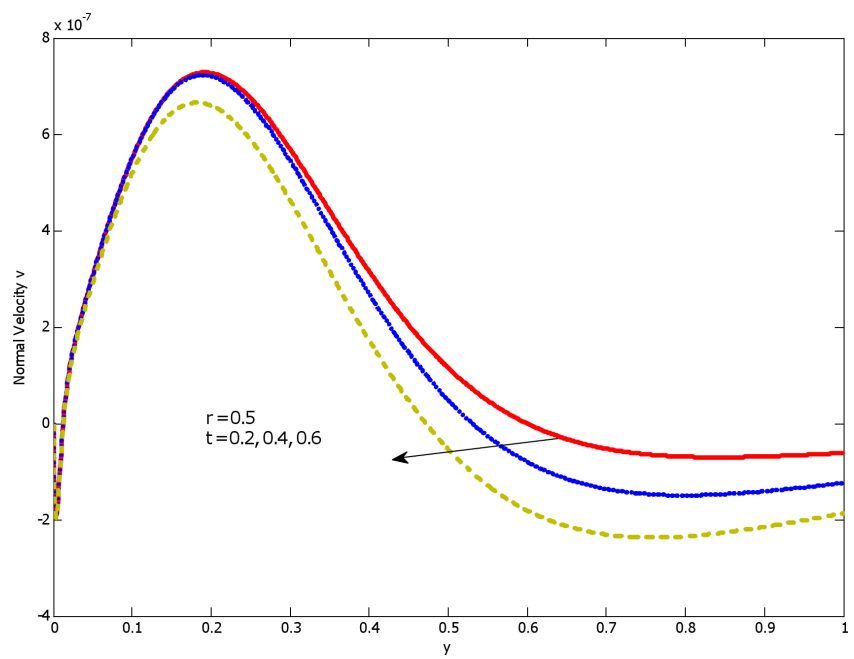

Fig. 11. Variation of the normal velocity $v(r, y, t)$ with $y$

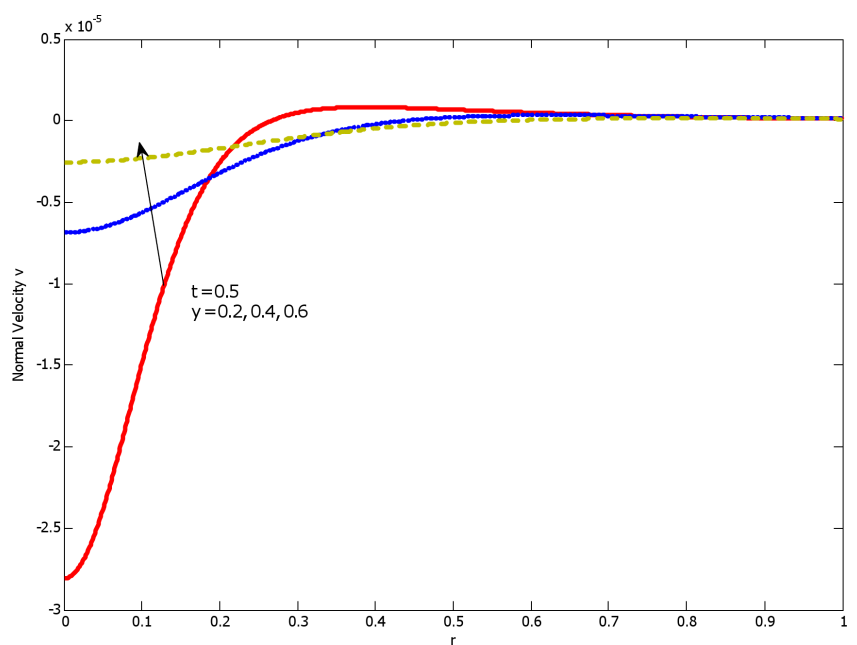

Fig. 12. Variation of the normal velocity $v(r, y, t)$ with $r$

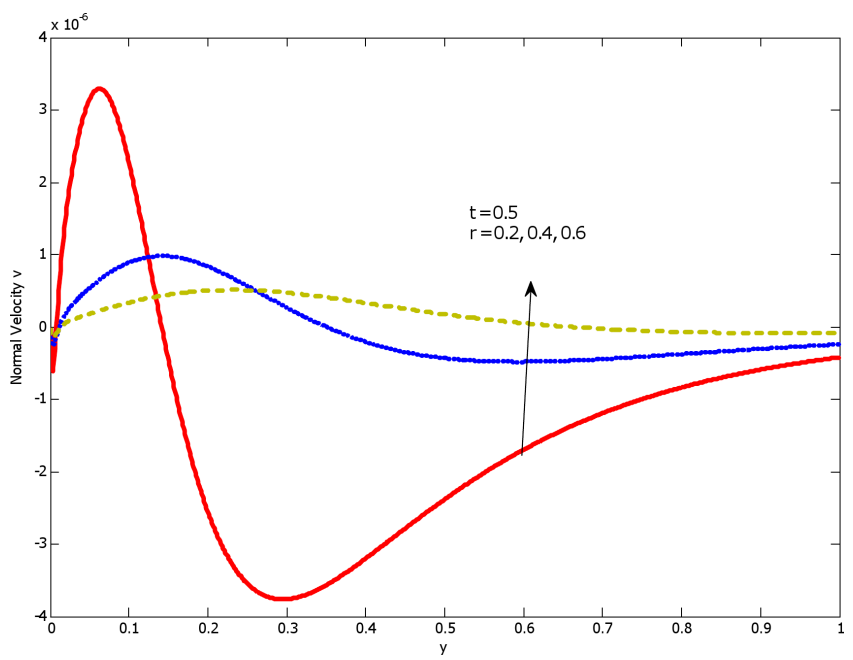

Fig. 13. Variation of the normal velocity $v(r, y, t)$ with $y$

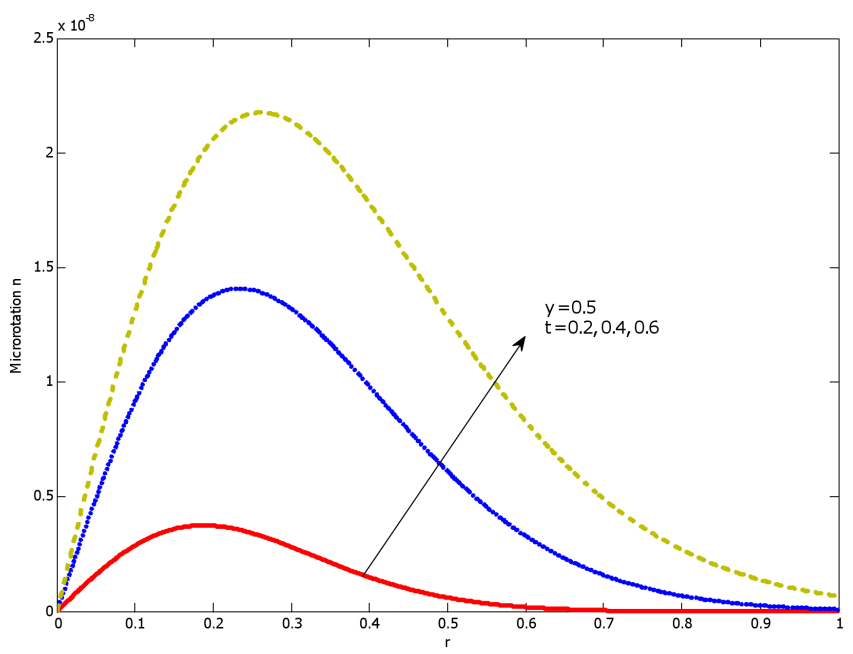

Fig. 14. Variation of the microrotation $n(r, y, t)$ with $r$

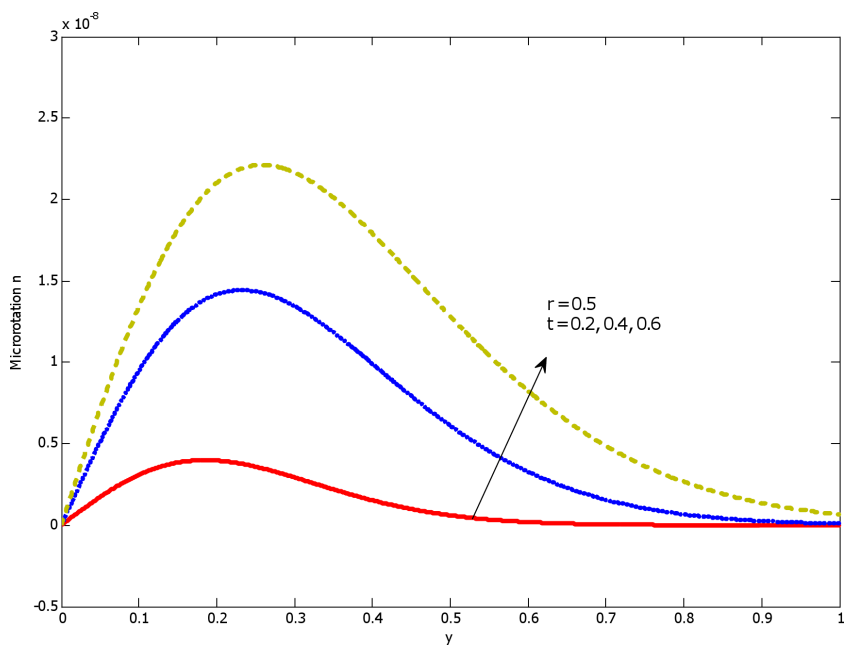

Fig. 15. Variation of the microrotation $n(r, y, t)$ with $y$ 
I.H. El-Sirafy and A.W. Aboutahoun

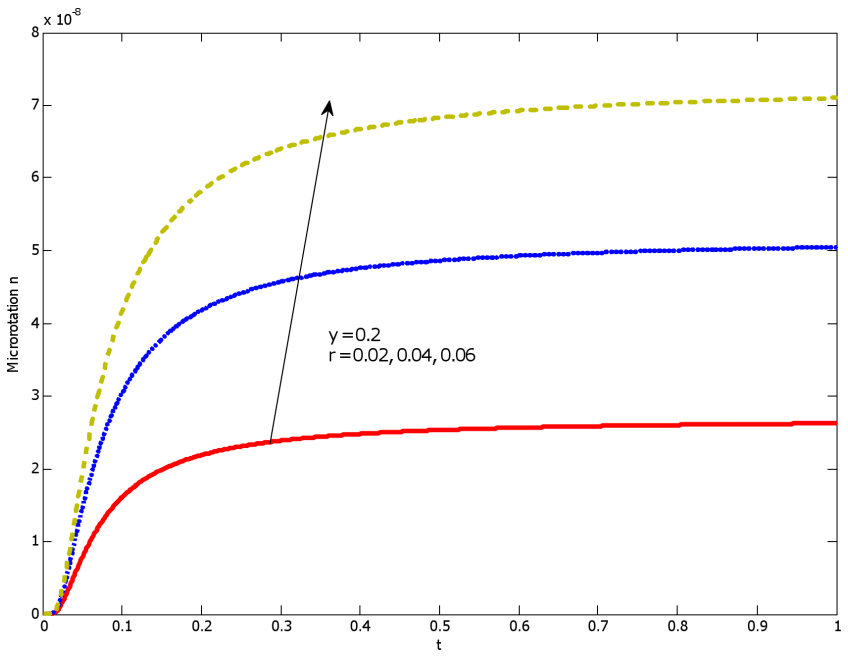

Fig. 16. Variation of the microrotation $n(r, y, t)$ with $t$

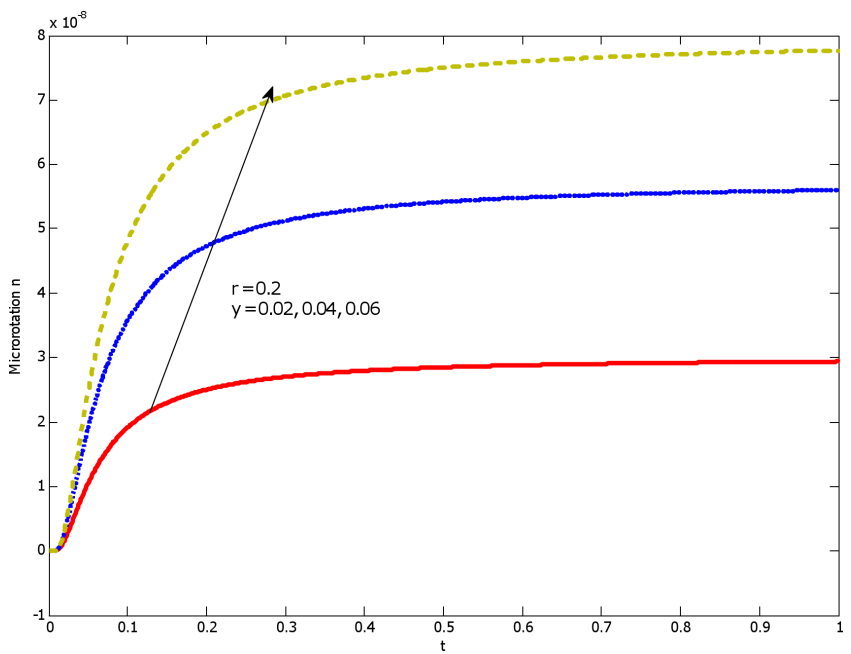

Fig. 17. Variation of the microrotation $n(r, y, t)$ with $t$

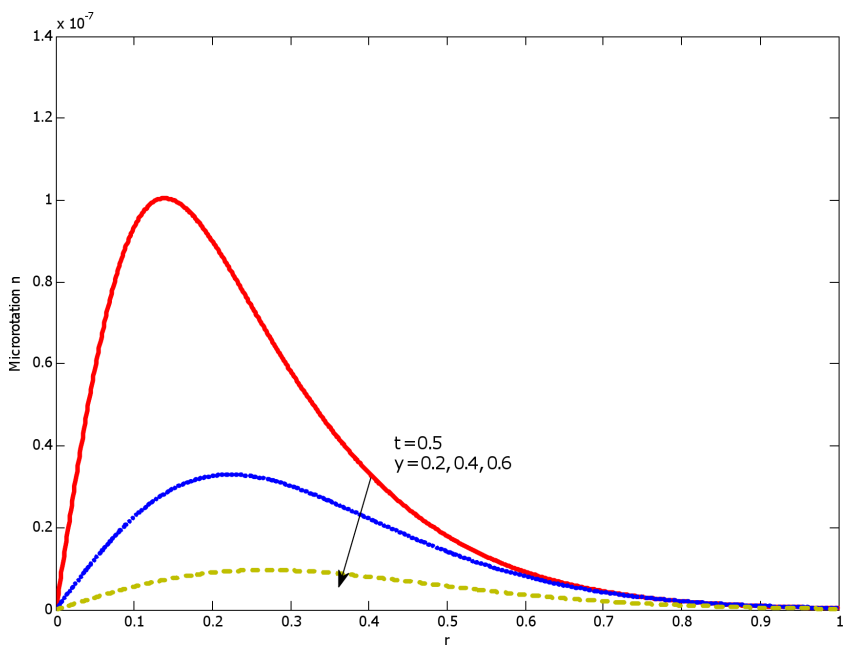

Fig. 18. Variation of the microrotation $n(r, y, t)$ with $r$

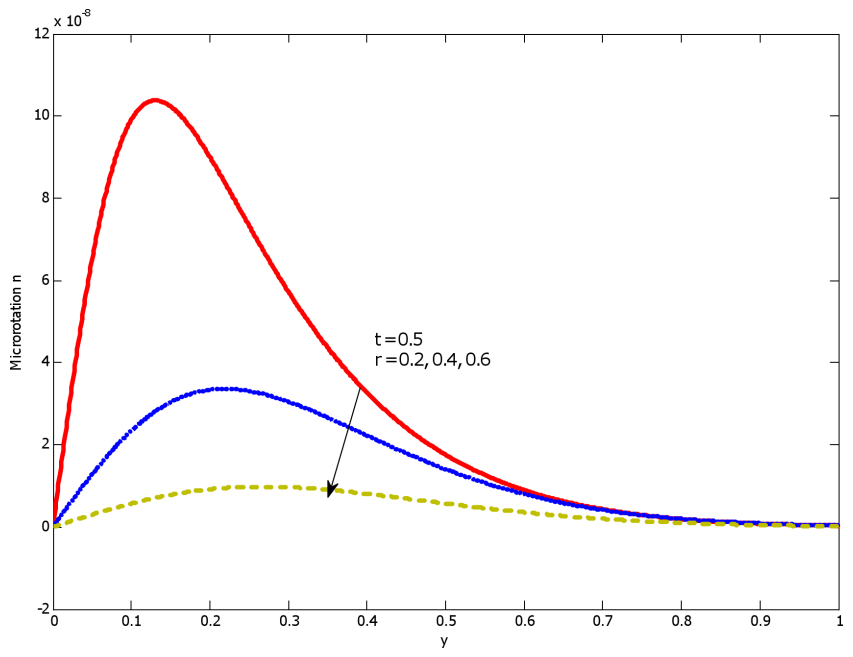

Fig. 19. Variation of the microrotation $n(r, y, t)$ with $y$

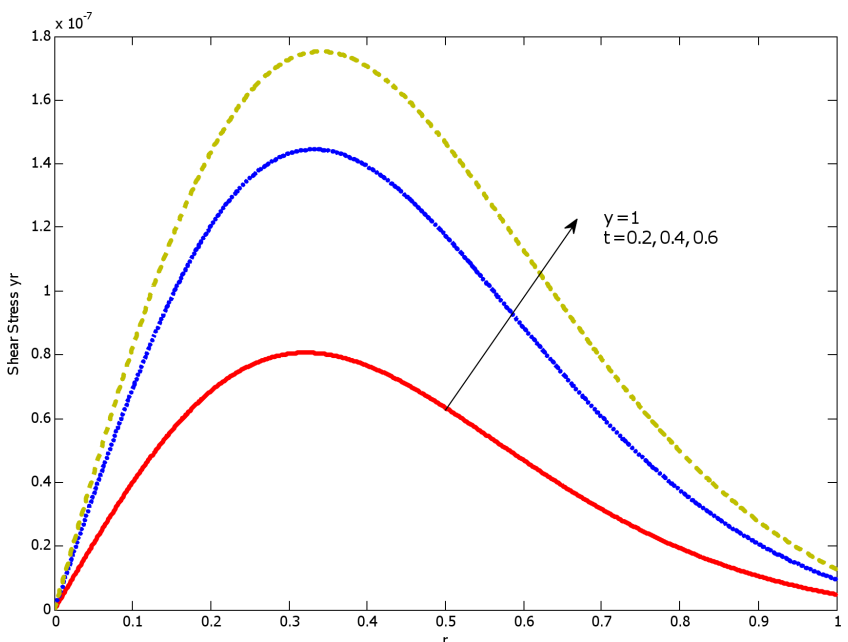

Fig. 20. Variation of the shear stress $\widehat{y r}$ with $r$

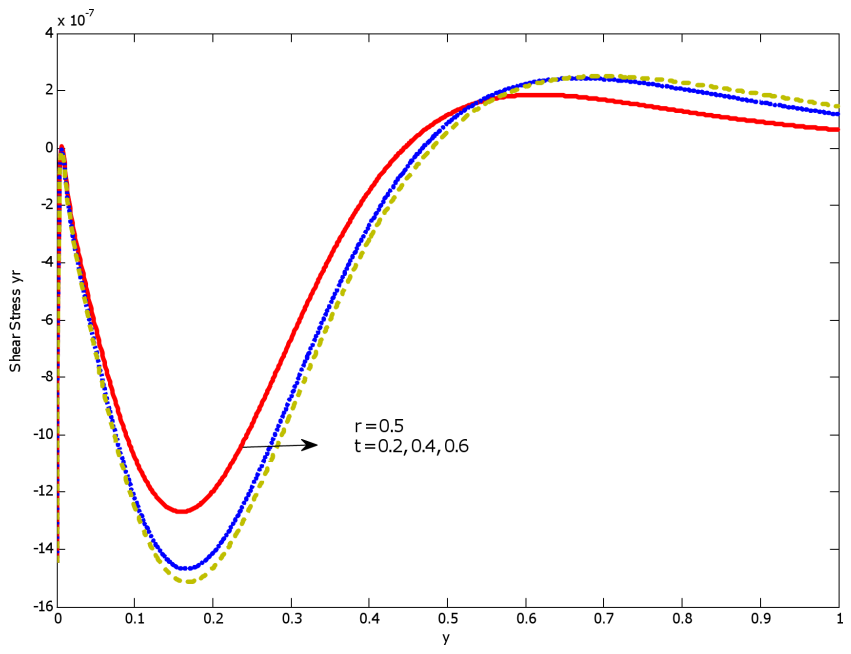

Fig. 21. Variation of the shear stress $\widehat{y r}$ with $y$ 
On axisymmetrical boundary problem of unsteady motion of micropolar fluid in the half-space

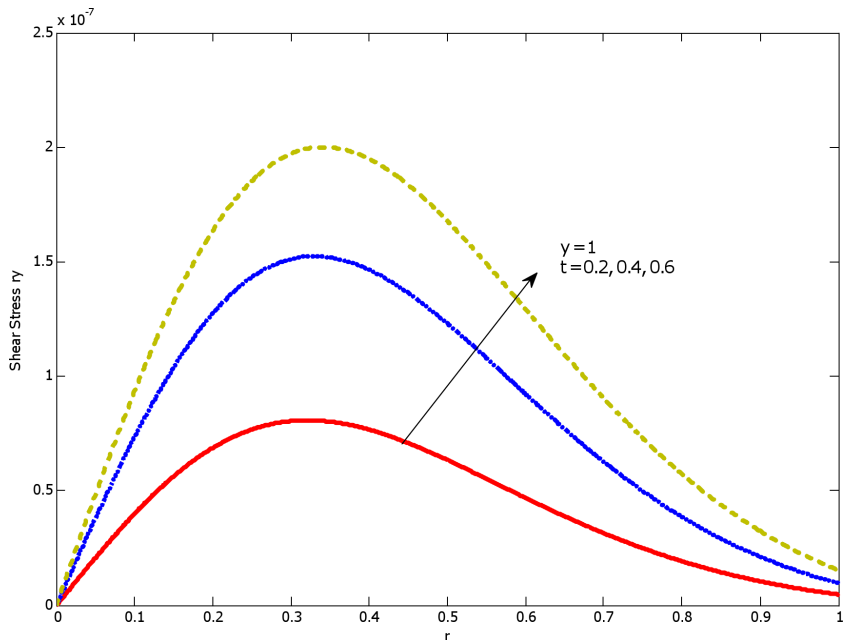

Fig. 22. Variation of the shear stress $\widehat{r y}$ with $r$

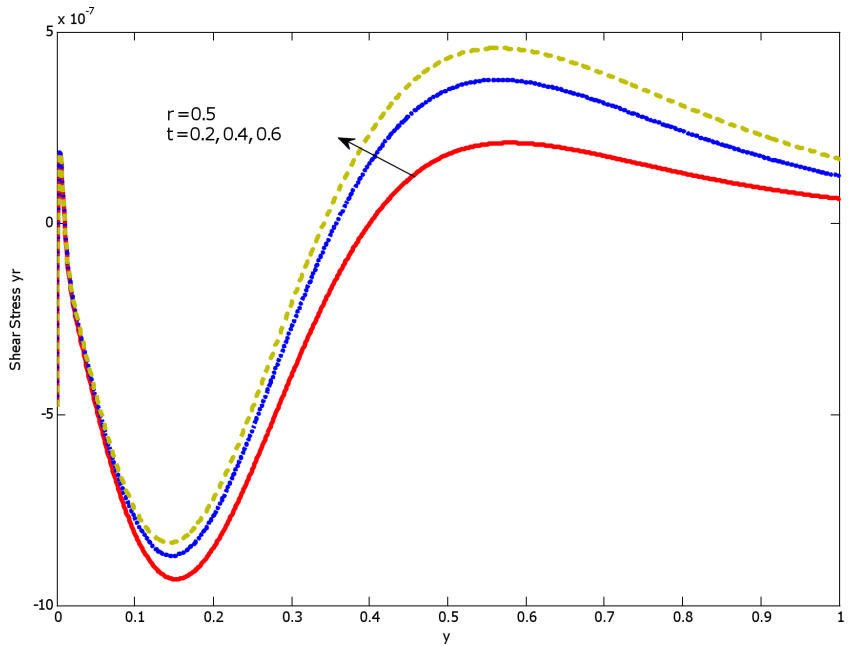

Fig. 23. Variation of the shear stress $\widehat{r y}$ with $y$

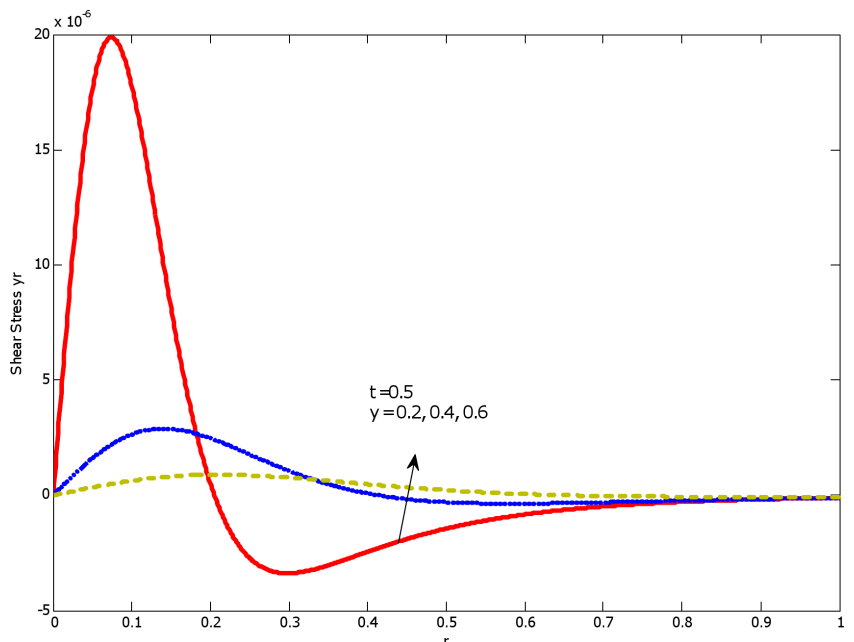

Fig. 24. Variation of the shear stress $\widehat{y r}$ with $r$

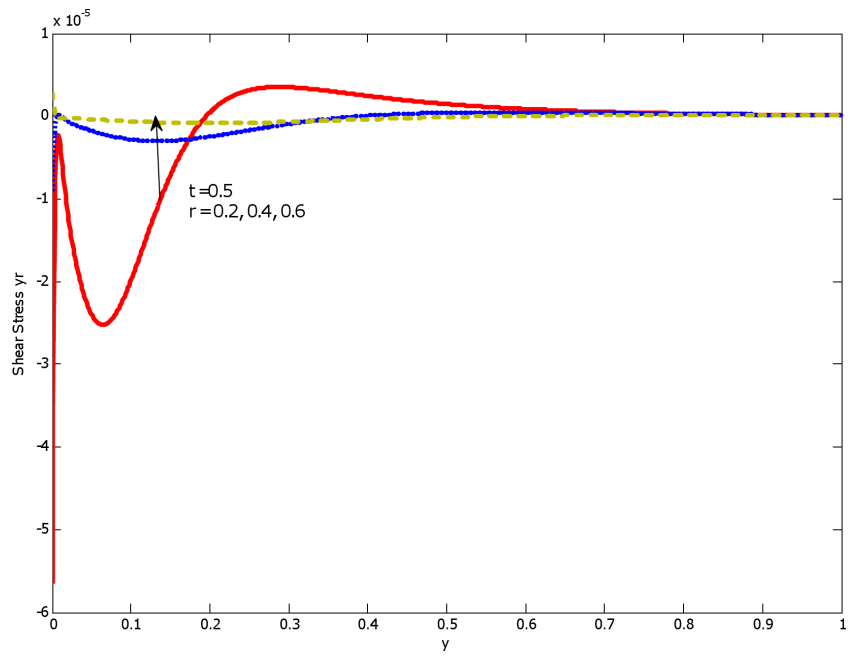

Fig. 25. Variation of the shear stress $\widehat{y r}$ with $y$

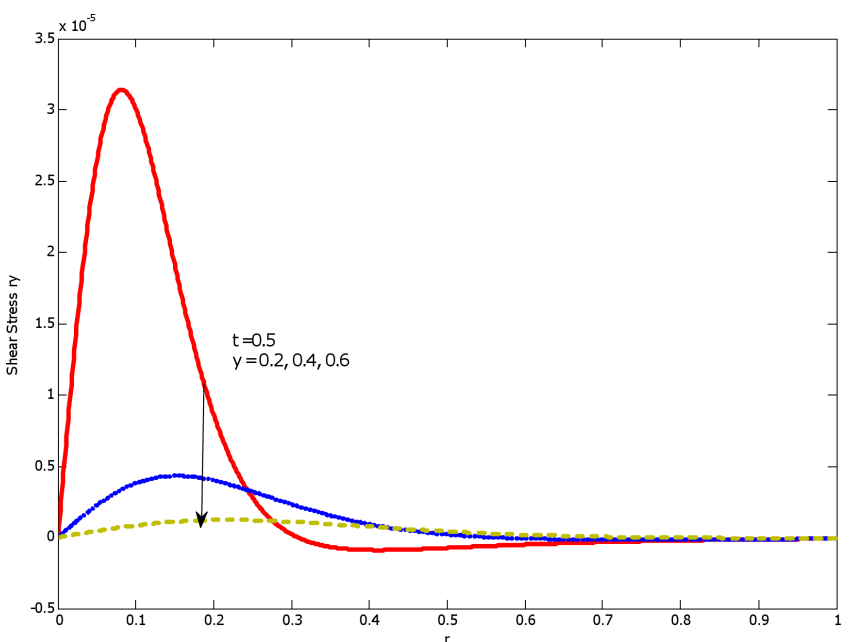

Fig. 26. Variation of the shear stress $\widehat{r y}$ with $r$

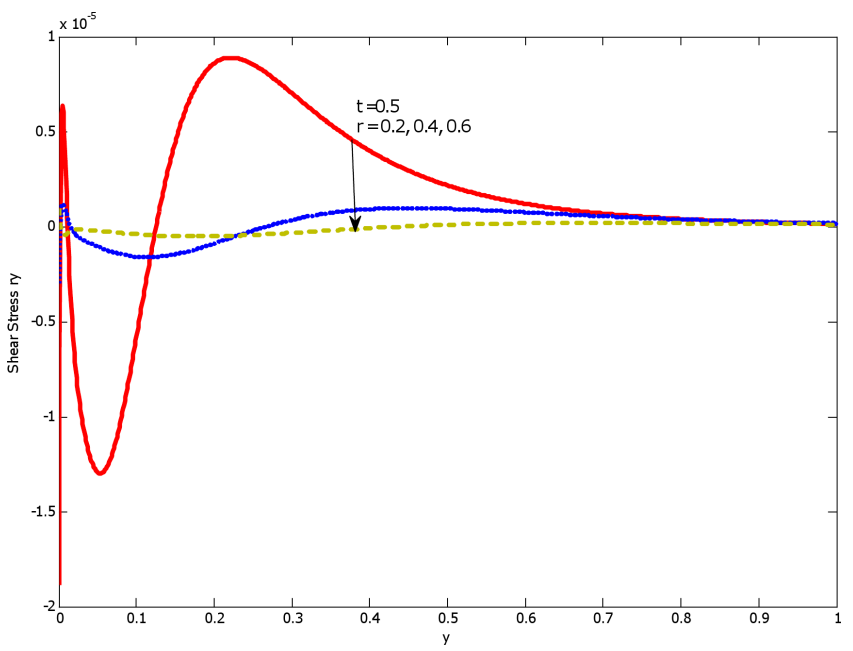

Fig. 27. Variation of the shear stress $\widehat{r y}$ with $y$ 
I.H. El-Sirafy and A.W. Aboutahoun

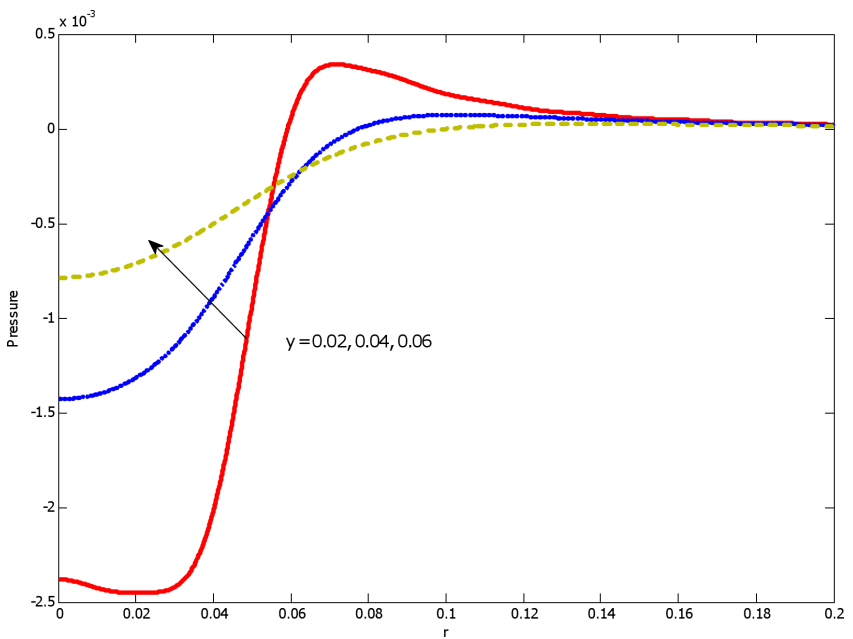

Fig. 28. Variation of the pressure $P(r, y)$ with $r$

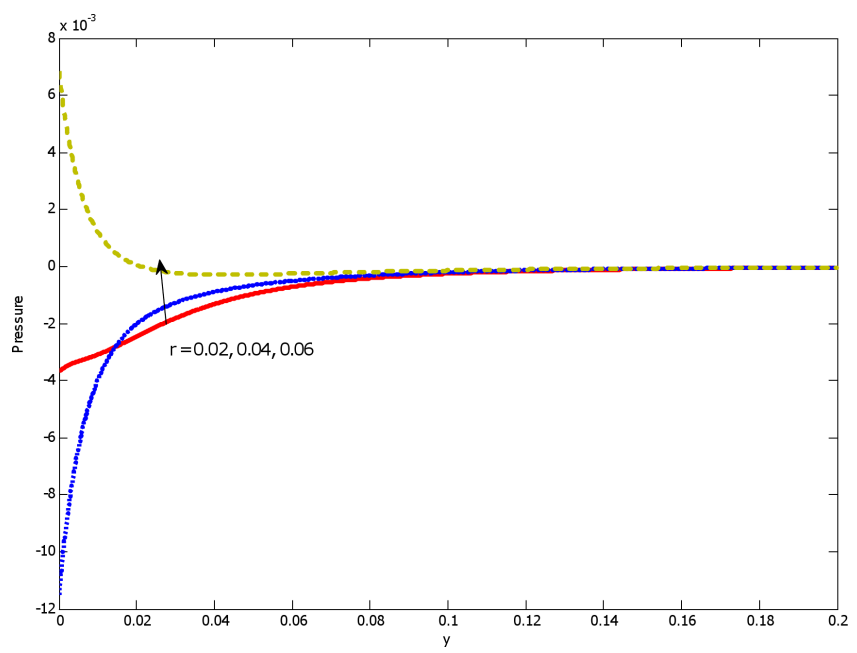

Fig. 29. Variation of the pressure $P(r, y)$ with $y$

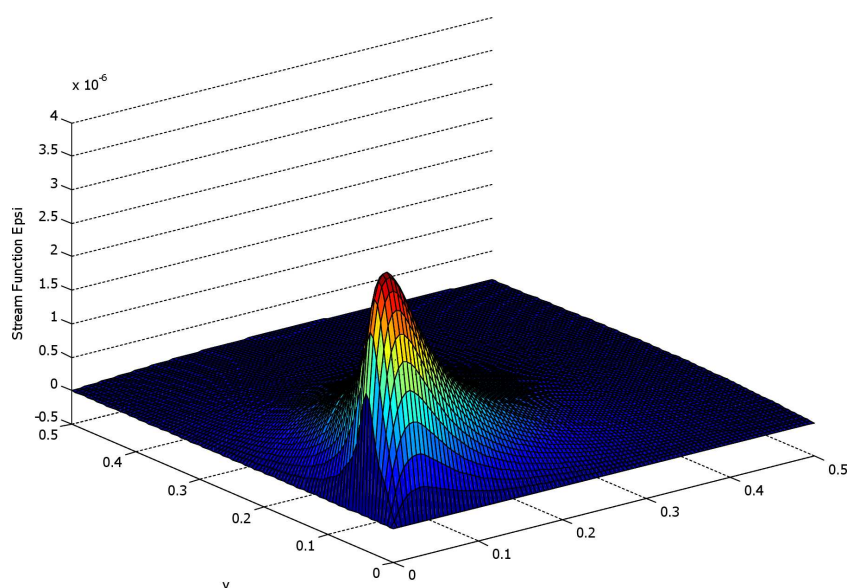

Fig. 30. Variation of the stream function $\Psi(r, y, t)$ with $r$ and $y$ when $t=0.5$

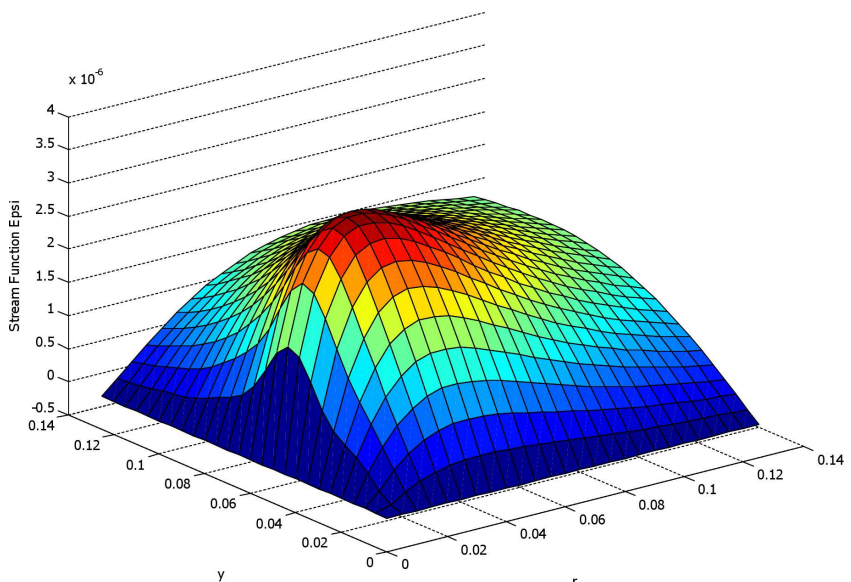

Fig. 31. Variation of the stream function $\Psi(r, y, t)$ with $r$ and $y$ when $t=0.5$

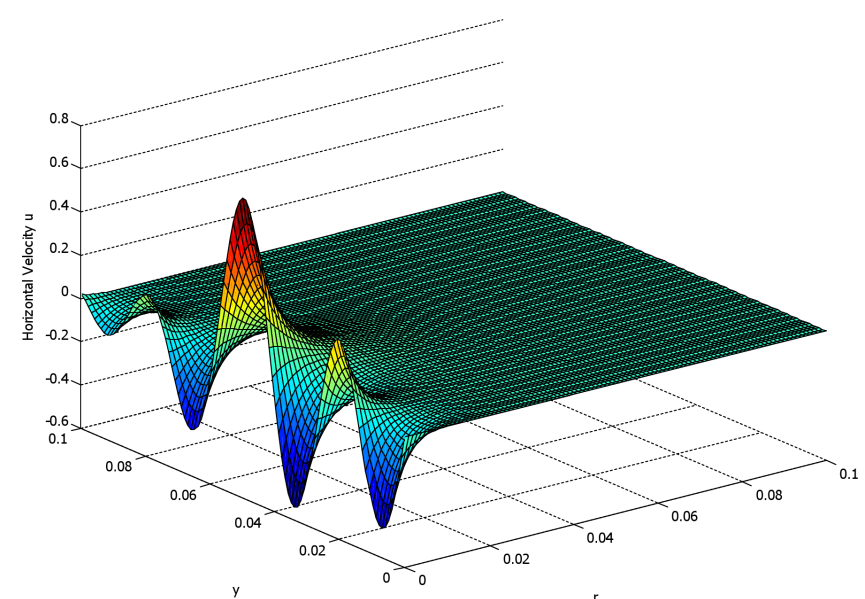

Fig. 32. Variation of the horizontal velocity $u(r, y, t)$ with $r$ and $y$ when $t=0.5$

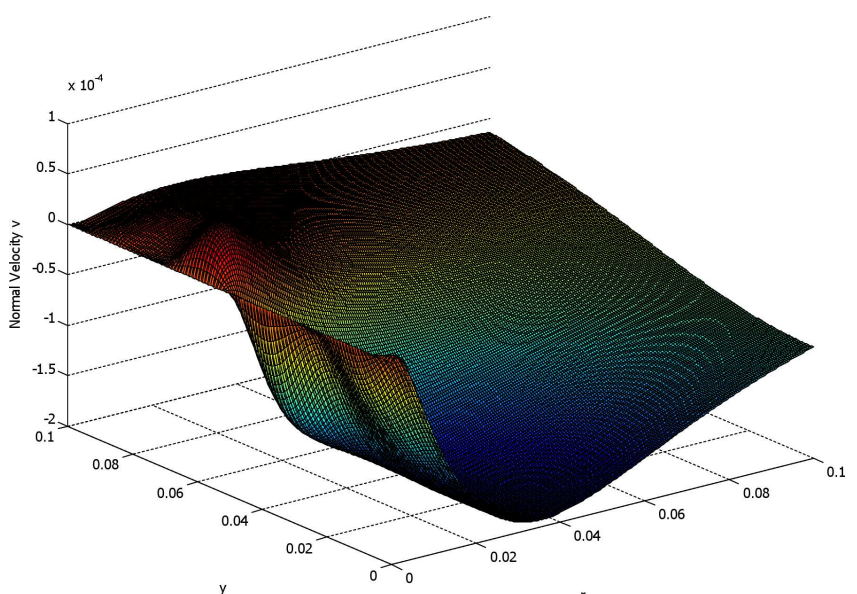

Fig. 33. Variation of the normal velocity $v(r, y, t)$ with $r$ and $y$ when $t=0.5$ 
On axisymmetrical boundary problem of unsteady motion of micropolar fluid in the half-space

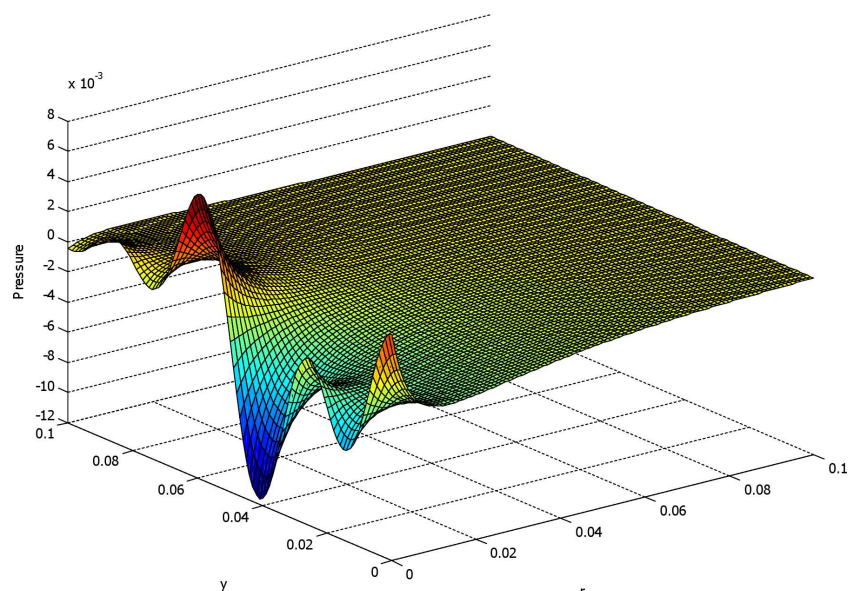

Fig. 34. Variation of the pressure $p(r, y)$ with $r$ and $y$

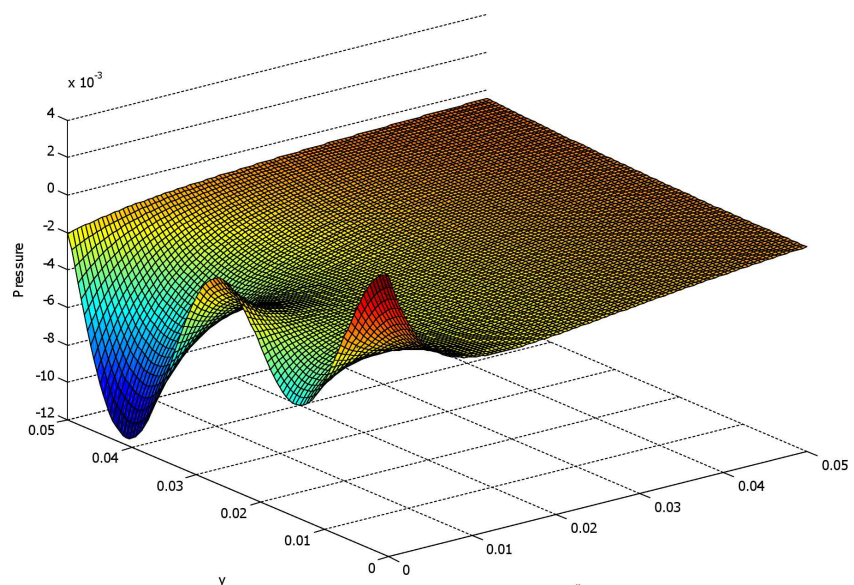

Fig. 35. Variation of the pressure $p(r, y)$ with $r$ and $y$

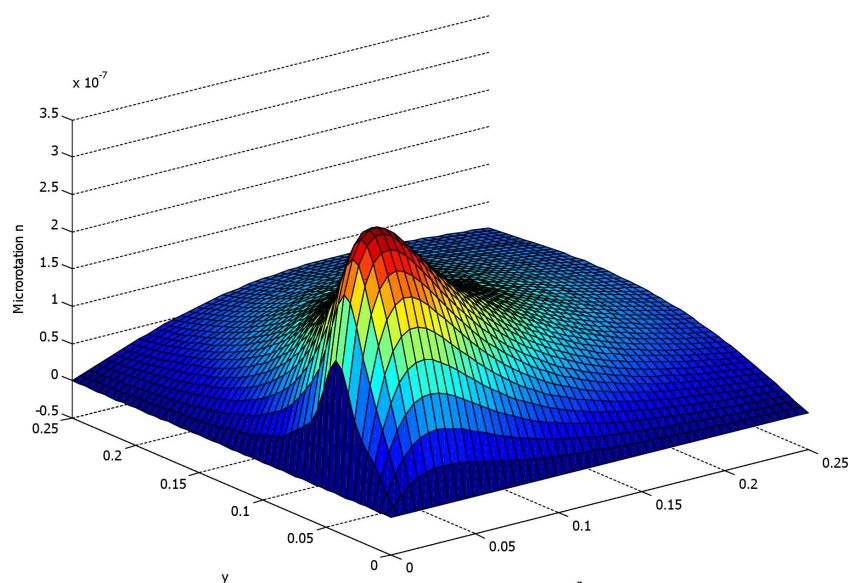

Fig. 36. Variation of the microrotation $n(r, y, t)$ with $r$ and $y$ when $t=0.5$

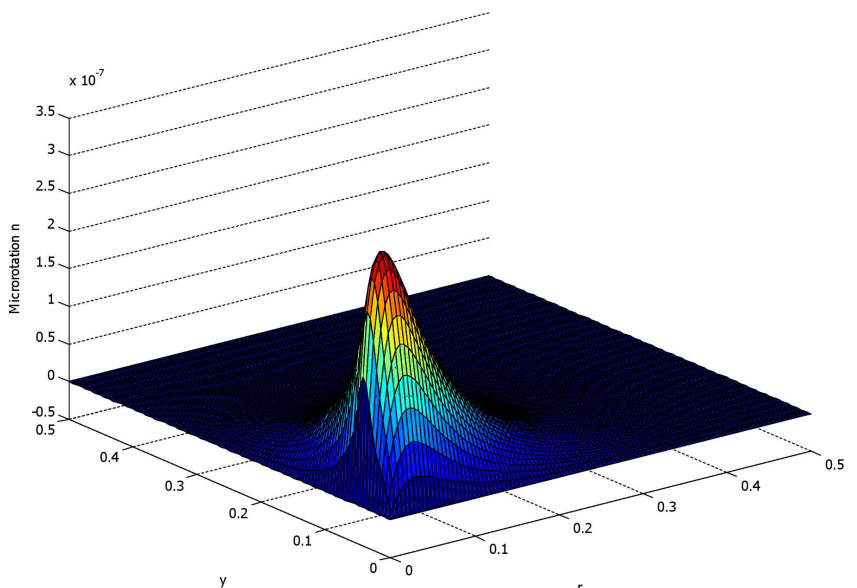

Fig. 37. Variation of the microrotation $n(r, y, t)$ with $r$ and $y$ when $t=0.5$

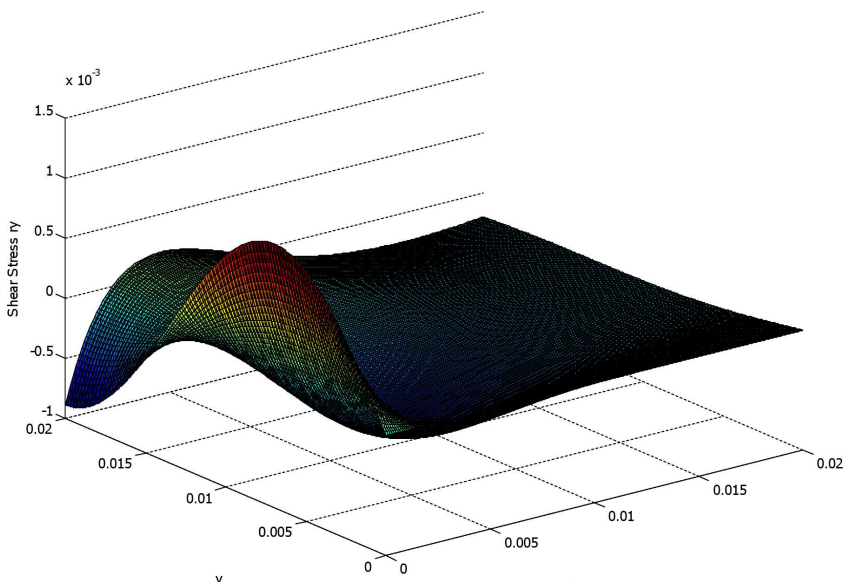

Fig. 38. Variation of the shear stress $\widehat{r y}(r, y, t)$ with $r$ and $y$ when $t=0.5$

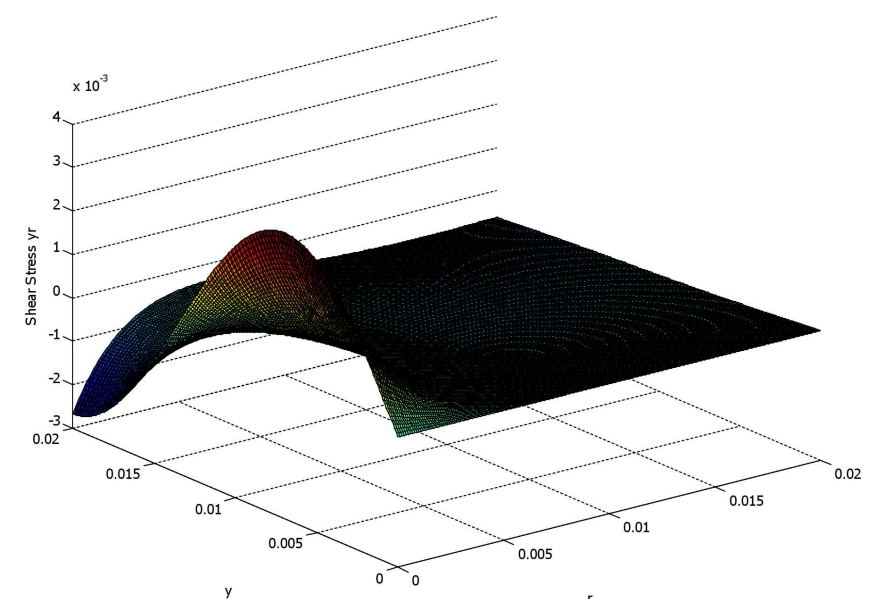

Fig. 39. Variation of the shear stress $\widehat{y r}(r, y, t)$ with $r$ and $y$ when $t=0.5$ 
I.H. El-Sirafy and A.W. Aboutahoun

\section{Conclusions}

The considered problem in this study is the corresponding problem to the two dimensional motion which is the axisymmetrical motion in the upper half-space. In this motion, the fluid particles move in planes passing through a certain line called the axis of symmetry and the motion in all such planes is identical. The problem of axisymmetrical flow of unsteady micropolar fluid in the half-space when the shear stresses are given on the boundary has been considered in this paper. An exact solution for this problem using the LaplaceHankel transform technique is obtained. Numerical results for the stream function, velocity components, microrotation, pressure, and shear stresses components are presented in graphs in two and three dimension spaces for various parameter conditions.

Acknowledgements. The authors wish to express their very sincere thanks to the anonymous referees for their valuable comments and suggestions.

\section{REFERENCES}

[1] A.C. Eringen, "Theory of micropolar fluids", J. Math. Mech. 16, 1-18 (1966).

[2] A.C. Eringen, Microcontinuum Field Theories I and II, Springer-Verlag, New York, 1998

[3] I.H. El-Sirafy, "Two-dimensional flow of nonstationary micropolar fluid in the half-plane for which the shear stresses are given on the boundary", J. Computational and Applied Mathematics 12 (13), 271-276 (1985).
[4] I.H. El-Sirafy and A.M. Abdel-Moneim, "Two-dimensional unsteady motion of micropolar fluid in the half-plane when the velocity are given on the boundary", IJRRAS 6 (3), 302-309 (2011).

[5] R.S. Gorla, M.A. Mansour, and A.A. Mohammedien, "Combined convection in an axisymmetric stagnation flow of micropolar fluid", Int. J. Num. Meth. Heat Fluid Flow 6 (4), 47-55 (1996).

[6] H. Bateman, Tables of Integral Transforms, vol. 1, McGrawHill, New York, 1954.

[7] M.S. Faltas, H.H. Sherief, and E.A. Ashmawy, "Interaction of two spherical particles rotating in a micropolar fluid", Mathematical and Computer Modelling 56 (9-10), 229-239 (2012).

[8] Y.Y. Lok, N. Amin, and I. Pop, "Unsteady mixed convection flow of a micropolar fluid near the stagnation point on a vertical surface", Int. J. Thermal Sciences 45, 1149-1157 (2006).

[9] K.A. Kline and S.J. Allen, "Nonsteady flows of fluids with microstructure", Physics of Fluids 13, 263-283 (1970).

[10] G. Lukaszewicz, Micropolar Fluids, Theory and Application, Birkhouser, Berlin, 1999.

[11] G. Ahmadi, "Self-similar solution of incompressible micropolar boundary layer flow over a semi-infinite flat plate", Int. J. Engineering and Science 14, 639-646 (1976).

[12] S.J. Allen and K.A. Kline, "Lubrication theory for micropolar fluids", Trans. A.S.M.E. J. Appl. Mech. 38, 646-650 (1971).

[13] D.A. Rees and A.P. Bassom, "The Blasius boundary layer flow of a micropolar fluid", Int. J. Engineering and Science 34, 113124 (1996).

[14] A. Kucaba-Piętal, "Microchannels flow modelling with the micropolar fluid theory", Bul. Pol. Ac.: Tech. 52 (3), 209-214 (2004). 